\title{
Investigação de Controle de Ordem Fracionária Aplicada ao Problema de Regulação de Velocidade de Turbina Hidráulica de 2kVA
}

\author{
Cláudia S. M. da Silva* Helen C. G. dos Reis* Nei J. da S. Farias* \\ Florindo A. de C. Ayres Júnior* Renan L.P. de Medeiros* Rafael da S. Mendonça* \\ * Departamento de Eletricidade, Faculdade de Tecnologia, Universidade Federal do Amazonas, Manaus-AM, Brasil \\ (e-mails: sabrinam901@gmail.com, helengomes.reis@gmail.com,neijunior1995@outlook.com, \\ florindoayres@ufam.edu.br,renanlandau@ufam.edu.br,mendonçarms@ufam.edu.br).
}

\begin{abstract}
In this paper, automatic control techniques are investigated, aiming to improving the performance of the speed governor, increasing the efficiency of the Electric Power System. For this, a linear model of a reduced-scale generation system is used to design, in a computational environment, a PID controller of fractional order (FOPID, based on an analytical method of calculating parameters from the specified margin values of gain and phase. The behavior of the FOPID controller will be presented through computational simulation using the MATLAB/Simulink tool, aiming to equate its performance with the PID controlled tuned by conventional methods.
\end{abstract}

Resumo: Neste artigo, são investigadas técnicas de controle automático, que visam a melhoria do desempenho do regulador de velocidade, aumentando a eficiência do Sistema Elétrico de Potência. Para isso, utiliza-se um modelo linear de um sistema de geração em escala reduzida para projetar, em ambiente computacional, um controlador PID de ordem fracionária (FOPID), fundamentado em um método analítico de cálculo de parâmetros a partir dos valores especificados de margem de ganho e fase. O comportamento do controlador FOPID será apresentado através de simulação computacional utilizando a ferramenta MATLAB/Simulink, buscando equiparar o seu desempenho com o controlador PID sintonizado por métodos convencionais.

Keywords: Automatic control; Speed governor; Electric Power System; FOPID; Computational simulation.

Palavras-chaves: Controle automático; Regulador de velocidade; Sistema Elétrico de Potência; FOPID; Simulação computacional.

\section{INTRODUÇÃO}

Com o crescimento populacional e econômico do Brasil, houve um aumento significativo do consumo de energia elétrica (Finkler et al. 2016). Para atender a demanda requisitada de potência gerada, necessita-se investir na construção de novas usinas e realização de melhorias nos sistemas já existentes.

Atualmente, a principal fonte de geração renovável de energia do Brasil é proveniente do movimento das águas e, com o aumento da complexidade do Sistema Elétrico de Potência e das tecnologias aplicadas a ele, surgiram diversos problemas relacionado a dinâmica das máquinas que compõem as hidrelétricas (Nascimento Filho, 2011).

Das máquinas que integram o sistema de geração de energia de usinas hidrelétricas, podemos destacar a turbina hidráulica, que é responsável pela conversão de energia cinética em energia mecânica, sendo o seu controle efetuado por meio do regulador de velocidade (RV) (Kundur, 1994).

Em Nascimento Filho (2011) são investigadas estratégias de controle digital aplicadas ao regulador de velocidade, sendo que, os estudos são implementados em uma micromáquina de 10kVA que modela um sistema físico real de geração de energia em escala reduzida. A partir dos subsistemas eletrônicos e computacionais desenvolvidos foi possível projetar um regulador de velocidade digital baseado na estrutura canônica RST de controle digital.

Com o proposito de melhorar o desempenho do regulador de velocidade e do sistema de geração de energia, Ayres Júnior et al. (2014) desenvolveu um controlador PID (ProporcionalIntegral-Derivativo) de ordem fracionária baseado em um método de margens de ganho e fase. Para isso, utilizou-se um sistema em escala reduzida de $10 \mathrm{kVA}$ para realizar a implementação e os testes dos controladores aplicados ao controle e regulação de velocidade (RV), avaliando o desempenho do controlador FOPID (Fractional-Order Proportional Integral Derivate) tanto por simulações computacionais, quanto por testes experimentais.

Neste trabalho, são investigadas técnicas de controle PID de ordem fracionária. Para isso, utiliza-se um sistema linearizado em escala reduzida para projetar três controladores PID na plataforma MATLAB/Simulink (Mathworks, 2019), pelos métodos de alocação polinomial de polos, lugar geométrico das raízes e margem de ganho e fase. Após o desenvolvimento dos controladores, foram realizados testes em um sistema não linear que permite reproduzir através de simulações o sistema de geração hidráulico.

\section{REVISÃO BIBLIOGRÁFICA}




\subsection{Funcionamento do Sistema de Energia de Usinas Hidrelétricas}

O sistema de geração de energia de usinas hidrelétricas, tem como princípio de funcionamento a transformação de energia cinética, oriunda da movimentação das águas, em energia mecânica na turbina hidráulica, deslocando as hélices da turbina e, consequentemente do eixo a qual encontra-se acoplada uma máquina síncrona, realizando assim a geração de energia elétrica (Kundur, 1994).

\subsection{Regulador de Velocidade}

O regulador de velocidade é um controlador que atua no circuito hidráulico, ele visa manter os geradores síncronos operando na velocidade síncrona, pois, devido as condições de operação do sistema de geração, essa velocidade possui variações. Sendo assim, o RV é responsável pelo controle da potência mecânica da turbina e do gerador, consequentemente da frequência elétrica que está sendo gerada, garantindo que esteja em sincronia com a frequência da rede elétrica (Rocha et al. 2012).

Dessa forma, necessita-se modernizar e automatizar o regulador de velocidade, pois, o processo por ele realizado é responsável por manter a frequência elétrica gerada em sincronia com a frequência da rede, promovendo a qualidade da energia elétrica.

\subsection{Controladores PID Convencionais}

O controlador PID projetado pelo método clássico, possui a função de transferência apresentada em (1). De acordo com Bazanella et al. (2005), a ação integrativa realiza a eliminação dos erros em regime permanente, a ação derivativa antecipa o comportamento do processo e a ação de proporcionalidade faz com que o sistema tenha uma reação rápida ao erro presente.

Projeta-se um sistema de controle para minimizar ou eliminar a ação das perturbações, fazendo com que ao se aplicar o sinal de controle, a saída apresente o comportamento desejado pelo projetista. Para que isso seja possível, é necessário saber qual o comportamento almejado na saída e conhecer as dinâmicas físicas relacionadas aos processos (Bazanella et al. 2005).

$$
C(s)=k_{p}+\frac{k_{I}}{s}+k_{D} s
$$

\subsection{Controlador PID de Ordem Fracionária}

O controlador FOPID possui sua metodologia baseada em derivadas e integrais de ordem fracionária, onde o mesmo possui cinco parâmetros de sintonização $\left(K_{P}, K_{I}, K_{D}, \lambda, e \mu\right)$, onde $\lambda$ refere-se a parte integrativa e $\mu$ a parte derivativa deste controlador (Valério et al. 2013).

$$
C(s)=k_{P}+\frac{k_{I}}{s^{\lambda}}+k_{D} s^{\mu}
$$

O FOPID apresenta uma região de ajustes de parâmetros mais ampla que o controlador PID convencional, ocasionando maior flexibilidade para o projeto, por isso, optou-se pela sua implementação (Ayres Junior et al. 2014).

\section{CONFIGURAÇÃO DO SISTEMA}

\subsection{Sistema de Geração de Energia em Escala Reduzida}

Para a realização das simulações em ambiente computacional, inicialmente, obteve-se a modelagem dinâmica do sistema primário de hidrogeração.

Em Kundur (1994) é desenvolvido um conjunto de equações não lineares apresentadas em (3), que representa o modelo matemático da turbina hidráulica de maneira simplificada, sendo, a água e as paredes do conduto forçado consideradas incompressíveis.

$$
\left\{\begin{array}{c}
\bar{G}=A_{t} \bar{g} \\
\bar{H}=\left(\frac{\bar{U}}{\bar{G}}\right)^{2} \\
\bar{U}=\frac{1}{T_{w} s}\left(\bar{H}_{o}-\bar{H}\right) \\
\overline{P_{m}}=\bar{H}\left(\bar{U}-\overline{U_{n l}}\right. \\
\overline{T_{m}}=\overline{P_{r}} \frac{\overline{P_{m}}}{\bar{\omega}}
\end{array}\right.
$$

onde, $\bar{G}$ é a abertura ideal do distribuidor; $A_{t}$ é o ganho da turbina hidráulica; $\bar{g}$ é a abertura de fato do distribuidor; $\bar{H}$ é a altura do lago; $\bar{U}$ é a velocidade da água na turbina; $\overline{U_{n l}}$ é a velocidade da água com a turbina rodando sem carga; $\bar{H}_{o}$ é a queda hidráulica inicial; $\overline{P_{m}}$ é a potência mecânica desenvolvida pela turbina; $\bar{\omega}$ é a velocidade do conjunto turbina-gerador; $\overline{T_{m}}$ é o torque mecânico desenvolvido pela turbina; $T_{w}$ é o tempo de partida da água e; $\bar{P}_{r}$ é a mudança de base.

$$
\overline{P_{r}}=\frac{\text { Potência nominal da Turbina }}{\text { Potência base do sistema }}
$$

Para a realização do projeto dos controladores é necessário utilizar a função de transferência da turbina hidráulica, para isso, lineariza-se as equações em torno do ponto de operação $\bar{U}_{o}, \bar{G}_{o}, \bar{H}_{o}$, e ${\overline{P_{m}}}_{o}$, obtendo-se a função de transferência (5). (Kundur, 1994; Nascimento Filho, 2011).

$$
G_{1}(s)=\frac{1-T_{\omega} s}{1+\frac{1}{2} T_{\omega} s}
$$

A função de transferência que representa a modelagem simplificada da válvula e do servomotor é desenvolvida em Kundur (1994) e apresentada em Nascimento Filho (2011), conforme exposto em (6).

$$
\frac{Y(s)}{X(s)}=\frac{K}{s}
$$

Sendo: $Y$ o deslocamento do pistão do servomotor; $X$ o deslocamento do carretel da válvula, que aciona o servomotor e; $K$ o ganho do conjunto válvula e servomotor. 
Também em Nascimento filho (2011), desenvolveu-se um modelo por espaço de estados do servoposicionador que é exposto em (7).

$$
\left\{\begin{array}{c}
{\left[\begin{array}{l}
\dot{x}_{1} \\
x_{2}
\end{array}\right]=\left[\begin{array}{cc}
0 & \frac{1}{t_{g}} \\
-\frac{1}{t_{p}} & -\frac{1}{t_{p}}
\end{array}\right]\left[\begin{array}{l}
x_{1} \\
x_{2}
\end{array}\right]+\left[\begin{array}{l}
0 \\
\frac{1}{t_{p}}
\end{array}\right] u} \\
g_{\min } \leq x_{1} \leq g_{\max } \\
y=\left[\begin{array}{ll}
1 & 0
\end{array}\right]\left[\begin{array}{l}
x_{1} \\
x_{2}
\end{array}\right]
\end{array}\right.
$$

onde, $u$ é o sinal de controle do regulador de velocidade e, os demais parâmetros são apresentados nas Tabelas 1, e 2 com seus respectivos valores.

Para projetar os controladores será utilizada a função de transferência simplificada (8), desenvolvido através do modelo de espaço de estados do servoposicionador mostrado em (7) considerando $t_{g} \ll t_{p}$ (Nascimento Filho, 2011).

$$
G_{2}(s)=\frac{1}{t_{g} s+1}
$$

A função de transferência do modelo de como o motor de corrente contínua será visto pelo sistema durante o processo de emulação é apresentada em (9) (Nascimento Filho, 2011).

$$
G_{3}(s)=\frac{1}{2 H s+B_{p u}}
$$

Os parâmetros da dinâmica da turbina hidráulica, do servoposicionador e do modelo motor de corrente contínua são apresentadas nas Tabelas 1,2, e 3, respectivamente. Os valores dos parâmetros da micromáquina utilizada foram obtidos através de ensaios realizados e apresentados em Nascimento Filho (2011) e Farias (2019).

Tabela 1 - Parâmetros da turbina hidráulica

\begin{tabular}{l|c|c|c}
\hline \multicolumn{2}{c|}{ Parâmetros } & Unidade & Valor \\
\hline $\begin{array}{l}\text { Abertura mínima do } \\
\text { distribuidor }\end{array}$ & $\boldsymbol{g}_{\boldsymbol{m i n}}$ & $\mathrm{S}$ & 0,06 \\
\hline $\begin{array}{l}\text { Abertura máxima do } \\
\text { distribuidor }\end{array}$ & $\boldsymbol{g}_{\boldsymbol{m a ́} \boldsymbol{x}}$ & $\mathrm{S}$ & 0,96 \\
\hline Ganho da turbina & $\boldsymbol{A}_{\boldsymbol{t}}$ & - & 1,11 \\
\hline $\begin{array}{l}\text { Queda hidráulica } \\
\text { nominal do lago }\end{array}$ & $\boldsymbol{H}_{\boldsymbol{o}}$ & p.u. & 1,0 \\
\hline $\begin{array}{l}\text { Tempo de partida da } \\
\text { água }\end{array}$ & $\boldsymbol{T}_{\boldsymbol{\omega}}$ & $\mathrm{S}$ & 1,41 \\
\hline $\begin{array}{l}\text { Velocidade da água } \\
\text { sem carga }\end{array}$ & $\boldsymbol{U}_{\boldsymbol{n} \boldsymbol{l}}$ & p.u. & 0,068 \\
\hline Mudança de base & $\boldsymbol{P}_{\boldsymbol{r}}$ & - & 1,0 \\
\hline
\end{tabular}

Tabela 2 - Parâmetros do servoposicionador

\begin{tabular}{c|c|c|c}
\hline \multicolumn{2}{c|}{ Parâmetros } & Unidade & Valor \\
\hline $\begin{array}{c}\text { Constante de tempo da } \\
\text { válvula piloto }\end{array}$ & $\boldsymbol{t}_{\boldsymbol{p}}$ & $\mathrm{s}$ & 0,016 \\
\hline $\begin{array}{c}\text { Constante de tempo da } \\
\text { válvula de distribuição }\end{array}$ & $\boldsymbol{t}_{\boldsymbol{g}}$ & $\mathrm{s}$ & 2,8 \\
\hline
\end{tabular}

Tabela 3 - Parâmetros do motor CC

\begin{tabular}{l|c|c|c}
\hline \multicolumn{2}{c|}{ Parâmetros } & Unidade & Valor \\
\hline $\begin{array}{l}\text { Indutância do } \\
\text { enrolamento de armadura }\end{array}$ & $\boldsymbol{L}_{\boldsymbol{a}}$ & $\mathrm{H}$ & 0,207 \\
\hline $\begin{array}{l}\text { Resistência do } \\
\text { enrolamento da armadura }\end{array}$ & $\boldsymbol{R}_{\boldsymbol{a}}$ & $\Omega$ & 1,54 \\
\hline $\begin{array}{l}\text { Momento de inércia do } \\
\text { motor }\end{array}$ & $\boldsymbol{H}$ & $\mathrm{S}$ & 0,68 \\
\hline Atrito viscoso & $\boldsymbol{B}_{\boldsymbol{p u}}$ & p.u. & 0,158 \\
\hline $\begin{array}{l}\text { Constante de força contra } \\
\text { eletromotriz }\end{array}$ & $\boldsymbol{n}$ & - & 0,88 \\
\hline
\end{tabular}

Portanto, para a parametrização dos controladores RV, obtémse uma função de transferência que representa sistema de hidrogeração em escala reduzida e, utilizando os valores de parâmetros das Tabelas 1, 2, e 3, adquirimos a planta (11). Essa planta engloba as dinâmicas rotacionais físicas da máquina síncrona, a função de transferência da turbina hidráulica e, a função de transferência do servoposicionador, sendo a saída do sistema a velocidade angular no rotor da máquina.

$$
\begin{gathered}
G(s)=G_{1}(s) \times G_{2}(s) \times G_{3}(s) \\
G(s)=\frac{-0,52521 s+0,37249}{s^{3}+1,89176+0,71287 s+0,05885}
\end{gathered}
$$

\subsection{Controladores PID}

Os valores dos parâmetros dos controladores RV foram obtidos utilizando três metodologias de sintonia de controladores, sendo elas: lugar geométrico das raízes (LGR), alocação polinomial de polos e, sintonia analítica para obtenção dos parâmetros do controlador a partir dos valores de margem de ganho e fase. As especificações para realizar a sintonia desses controladores são definidas na Tabela 4.

Tabela 4 - Especificações dos controladores

\begin{tabular}{l|c|c|c}
\hline \multicolumn{2}{c|}{ Especificações } & Unidade & Valor \\
\hline Tempo de acomodação & $\boldsymbol{t}_{\boldsymbol{s}}$ & $\mathrm{S}$ & 45 \\
\hline Máximo sobressinal & $\boldsymbol{M}_{\boldsymbol{s} \boldsymbol{s}}$ & - & 0,1 \\
\hline $\begin{array}{l}\text { Fator de } \\
\text { amortecimento }\end{array}$ & $\boldsymbol{\zeta}$ & - & 0,5912 \\
\hline Frequência de natural & $\boldsymbol{\omega}_{\boldsymbol{n}}$ & $\mathrm{rad} / \mathrm{s}$ & 0,1126 \\
\hline Margem de ganho & $\boldsymbol{M}_{\boldsymbol{g}}$ & $\mathrm{dB}$ & 6 \\
\hline Margem de fase & $\boldsymbol{M}_{\boldsymbol{p}}$ & $\mathbf{0}$ & 65 \\
\hline $\begin{array}{l}\text { Frequência de } \\
\text { cruzamento de ganho }\end{array}$ & $\boldsymbol{\omega}_{\boldsymbol{c g}}$ & $\mathrm{rad} / \mathrm{s}$ & 0,1126 \\
\hline $\begin{array}{l}\text { Frequência de } \\
\text { cruzamento de fase }\end{array}$ & $\boldsymbol{\omega}_{\boldsymbol{c f}}$ & $\mathrm{rad} / \mathrm{s}$ & 0,5631 \\
\hline
\end{tabular}

O método baseado no lugar geométrico das raízes trata-se de uma ferramenta gráfica que permite visualizar a variação dos polos de um sistema em malha fechada a medida em que se altera o valor de um parâmetro específico que, geralmente, trata-se do ganho (Bazanella et al. 2005). 
Pelo método LGR, desenvolveu-se um controlador PID convencional, para isso, utilizou-se o comando rlocus do MATLAB, que plota o gráfico do root locus do sistema dinâmico em laço aberto. Esse comando fornece a trajetória dos polos de um sistema em malha fechada em função do ganho $k$ de realimentação, portanto, utilizam-se esses gráficos para analisar os efeitos da variação dos ganhos (Mathworks, 2019). Logo, usando (1) juntamente com a planta (11), constituiu-se o gráfico do root locus e, em seguida obteve-se os valores de $k$ apresentado em (1), de acordo com as especificações da Tabela 4.

Quando sabemos as posições desejadas dos polos do sistema em malha fechada para que o sinal de saída do sistema apresente a resposta almejada, utiliza-se a metodologia conhecida como alocação polinomial de polos e determina-se a função de transferência do controlador para que esses polos de laço fechado assumam os valores pré-estabelecidos. Neste caso, usa a equação diofantina apresentada em Bazanella et al. (2005) para obter esses coeficientes.

Portanto, desenvolveu-se a equação diofantina, com o ganho $\mathrm{K}=5$, em ambiente computacional e determinou-se a função de transferência do controlador convencional certificando que, juntamente com a planta (11), em laço fechado o sistema contenha as especificações desejadas.

O método analítico de sintonia de controladores baseado em margem de ganho e fase que é utilizado para se obter os parâmetros do controlador FOPID, a partir dos valores de margem de fase e ganho desejados em malha fechada Valério et al. (2013). Porém, para que o sistema possua número de incógnitas igual ao número de equações, o projetista optou por utilizar um caso particular onde define-se que $\lambda=1$, e $\mu$ um valor fracionário a ser encontrado, prevalecendo apenas quatro incógnitas $K_{P}, K_{I}, K_{D}, e \mu$ e quatro equações não-lineares apresentadas em Valério et al. (2013) (Ayres Junior et al. 2014).

Utiliza-se o método de numérico de Newton, com o intuito de realizar o cálculo por meio de iterações. O Método de Newton realiza a combinação de linearização e iteração nas aproximações numéricas. Portanto, a resultado das equações define parâmetros do controlador de ordem fracionária $K_{P}, K_{I}, K_{D}, e \mu$.

\subsection{Método de Aproximação de Oustaloup}

Visando a aplicação do controlador fracionário sintonizado, o mesmo foi aproximado utilizando o método de aproximação de $s^{\alpha}$, onde $\alpha$ pode ser $\lambda$ ou $\mu$, mais utilizada é o de Oustaloup, também chamado de aproximação de Crone, sendo que o mesmo consiste em funções de transferência de tempo contínuo (Valério et al. 2013). Esta aproximação é baseada no ajuste da curva de resposta, ou seja, realiza-se a implementação dentro de uma largura de banda limitada (São Roque, 2015), representado por uma rede de polos e zeros. Conforme exposto de (12) a (17).

$$
s^{q}=k \prod_{n=1}^{n=k} \frac{\left(1+\frac{s}{\omega_{z n}}\right)}{\left(1+\frac{s}{\omega_{p n}}\right)}, \text { para } \mathrm{q}>0
$$

$$
\begin{gathered}
\alpha=\left(\frac{\omega_{h}}{\omega_{l}}\right)^{\frac{q}{N}} \\
\eta=\left(\frac{\omega_{h}}{\omega_{l}}\right)^{\frac{(1-q)}{N}} \\
\omega_{z 1}=\omega_{l} \sqrt{\eta} \\
\omega_{z n}=\omega_{p, n-1} \eta, \operatorname{para} n=2, \ldots, N . \\
\omega_{p n}=\omega_{z, n-1} \alpha, \text { para } n=1, \ldots, N .
\end{gathered}
$$

Sendo: $q$ o fator fracionário; $n$ a quantidade de polos e zeros; o ganho $k$ é ajustado para que a aproximação possua ganho unitário na frequência de $1 \mathrm{rad} / \mathrm{s} ; \omega_{z n}$ frequência do zero em $n ; \omega_{p n}$ frequência do polo em $n ; \alpha$ e $\eta$ são equações auxiliares para serem aplicadas na aproximação do polo e do zero; $\omega_{z 1}$ primeiro zero da aproximação; $\omega_{l}$ baixa frequência na aproximação; e $\omega_{h}$ alta frequência na aproximação.

Desta forma, após a obtenção dos parâmetros do controlador FOPID utilizando as equações não-lineares, aplica-se a aproximação de Oustaloup, obtendo-se uma função aproximada de um derivador de ordem fracionária, pois, de acordo com o exposto em (2), $s^{\mu}$ representa a parte derivativa do controlador FOPID. A função que implementa o método de aproximação de Oustaloup em ambiente computacional, a ousta_fod, é apresentada em (Monje et al. 2010).

\section{RESULTADOS E DISCUSSÕES}

\subsection{Sintonia dos Controladores}

O controlador obtido pelo método de lugar geométrico é apresentado em (18) e o via alocação de polos em (19).

$$
\begin{gathered}
C_{1}(s)=0,19+\frac{0,01}{s}+0,00197 s \\
C_{2}(s)=\frac{-0,3823 s^{3}-0,1252 s^{2}+0,01352 s+0,0038}{s^{3}+0,5497 s^{2}+0,3648 s}
\end{gathered}
$$

Após a aplicação da técnica de sintonia do controlador FOPID, com uma tolerância desejada do método numérico de Newton menor que $10^{-8}$, os valores dos parâmetros obtidos do controlador FOPID são apresentados na Tabela 5. Em seguida, implementou-se o método de aproximação de Oustaloup para os parâmetros apresentados na Tabela 6 .

\section{Tabela 5 - Parâmetros obtidos para o controlador FOPID}

\begin{tabular}{c|c}
\hline Parâmetros & Valor \\
\hline$K_{P}$ & 0,1702 \\
\hline$K_{I}$ & 0,0185 \\
\hline$K_{D}$ & 0,1661 \\
\hline$\mu$ & 0,8145 \\
\hline
\end{tabular}


Tabela 6 - Valores dos parâmetros para implementação no método Oustaloup.

\begin{tabular}{l|c|c|c}
\hline \multicolumn{2}{c|}{ Parâmetros } & Unidade & Valor \\
\hline Frequência baixa & $\boldsymbol{w}_{\boldsymbol{b}}$ & $\mathrm{rad} / \mathrm{s}$ & 0,0398 \\
\hline Frequência alta & $\boldsymbol{w}_{\boldsymbol{h}}$ & $\mathrm{rad} / \mathrm{s}$ & 0,3185 \\
\hline $\begin{array}{l}\text { Ordem de } \\
\text { aproximação }\end{array}$ & $\boldsymbol{N}$ & - & 4 \\
\hline $\begin{array}{l}\text { Ordem do } \\
\text { diferenciador }\end{array}$ & $\boldsymbol{\mu}$ & - & 0,8145 \\
\hline
\end{tabular}

O Controlador equivalente Contínuo obtido é dado em (20):

$$
C_{3}(s)=\frac{0,2356 s^{5}+0,158 s^{4}+0,0408 s^{3}+5,065.10^{-3} s^{2}+3,031.10^{-4} s}{s^{5}+0,6551 s^{4}+0,1444 s^{3}+0,01269 s^{2}+3,751.10^{-4} s}
$$

\subsection{Diagramas de Aproximação e Simulação não linear}

A Fig. 1 apresenta os diagramas de Bode do controlador FOPID obtido a partir da aproximação de Oustaloup, para as frequências entre $10^{-2}$ e $10^{2}(\mathrm{rad} / \mathrm{s})$, onde o controlador fracionário foi implementado para três oitavas de largura de banda, sendo uma oitava e meia $(1,5)$ acima e abaixo da frequência de projeto (frequência natural - $\boldsymbol{\omega}_{\boldsymbol{n}}$ ) para as frequências (baixa $\omega_{b}$ e alta $\omega_{h}$ ). Observa-se que para as frequências compreendidas na faixa de aproximação e para frequências abaixo da faixa, os valores estão muito próximos, tanto em magnitude quanto em fase.
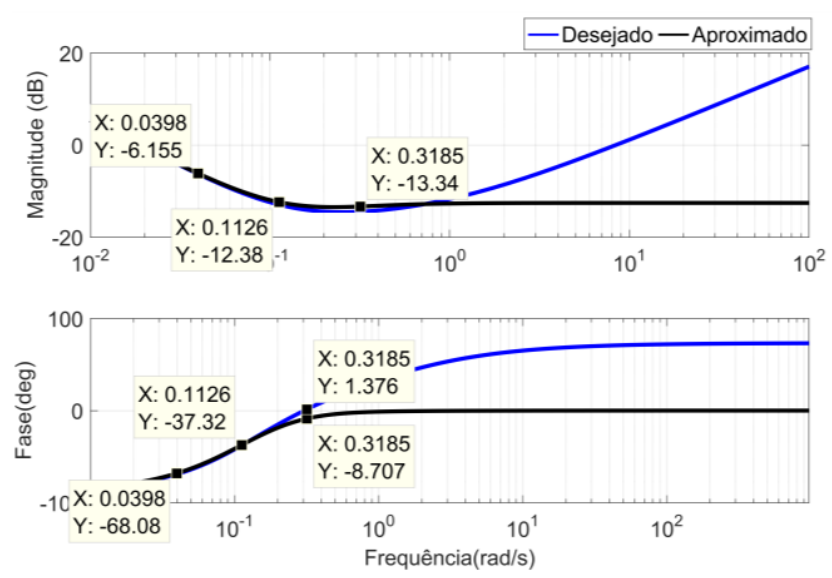

Fig. 1. Diagrama de Bode do controlador FOPID

Os três controladores projetados em um sistema linearizado em escala reduzida são constituídos na plataforma Simulink para realização de testes, sendo que, esses testes são realizados para o sistema não linearizado, de acordo com o diagrama de bloco apresentado na Fig. 2.

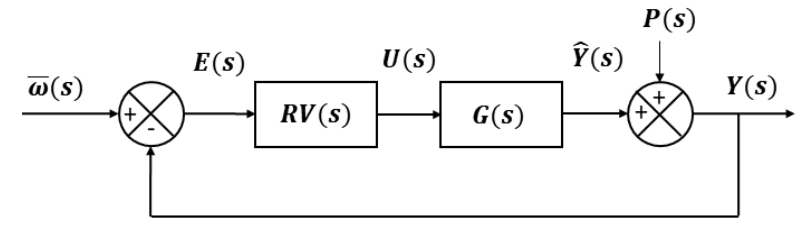

Fig. 2. Diagrama de blocos com dinâmica e controlador onde, $\bar{\omega}(s)$ é a referência de velocidade; $E(s)$ é o erro existente; $U(s)$ é o sinal de controle; $\widehat{Y}(s)$ é o sinal de saída; $Y(s)$ é o sinal de medida; $P(s)$ é a Perturbação de carga.

\subsection{Simulação com Variação do Sinal de Entrada}

Inicialmente, aplica-se um degrau unitário (1 p.u.) na entrada do sistema e após sua acomodação, efetuam-se dois testes. $\mathrm{O}$ primeiro teste visa realizar a variação de degrau na referência de velocidade.

A Fig. 3 representa a saída do sistema em escala reduzida quando realizado a variação de velocidade. Portanto, acrescenta-se um degrau com amplitude de 0,1 p.u. e, observase a aparição de um undershoot no controlador projetado via alocação de polos mais acentuado em comparação as demais metodologias comparadas.

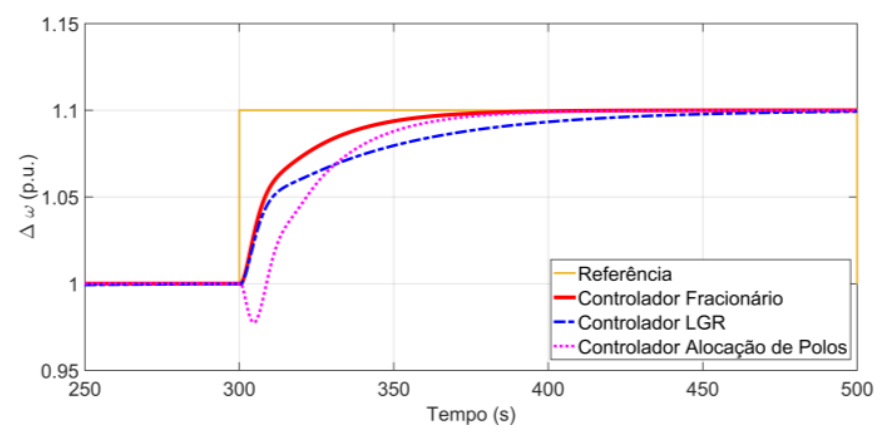

Fig. 3. Variação do sinal de entrada, inserção de velocidade.

Na Fig. 3, nota-se que nenhum dos controladores apresentou overshoot, porém, o controlador FOPID desenvolveu um sistema criticamente amortecido sem muitas distorções e com um tempo de acomodação menor, porém, vale ressaltar que o controlador projetado via alocação de polos proporcionou um undershoot acentuado em comparação as demais metodologias de projeto de controladores.

Para o primeiro momento do teste de variação de velocidade, o sinal de controle pode ser visualizado na Fig. 4. Observe que, o controlador via LGR sofre saturação no instante que é realizada a alteração de velocidade e, o controlador projetado pelo método de alocação de polos tem uma resposta inversa de aproximadamente 0,05 p.u. O controlador FOPID possui uma resposta suave, sem variações bruscas e um tempo de acomodação satisfatório.

Ainda no primeiro teste, após um período de 200 segundos, realiza-se a inserção de um sinal de entrada negativo de 0,1 p.u. fazendo com que o sistema retorne a sua condição inicial de regime permanente e, como pode ser visualizado na Fig. 5, o controlador PID de ordem fracionária é o que possui a melhor resposta, sendo, o seu tempo de acomodação menor que os outros controladores PID convencionais, obtendo-se uma declinação sem variações e distorções. O controlador via alocação de polos apresentou um overshoot no instante que foi realizada variação de velocidade, porém, obteve um tempo de acomodação semelhante ao do controlador FOPID, mas menor que o controlador LGR. 


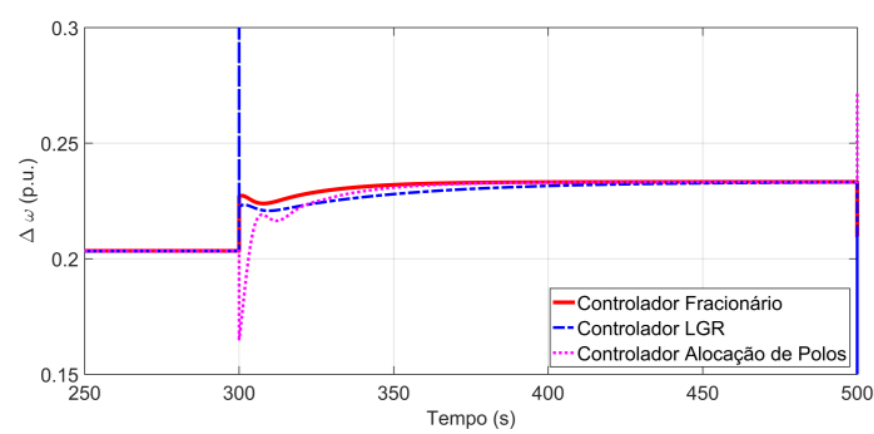

Fig. 4. Resposta do controlador a inserção de 0,1 p.u de velocidade.

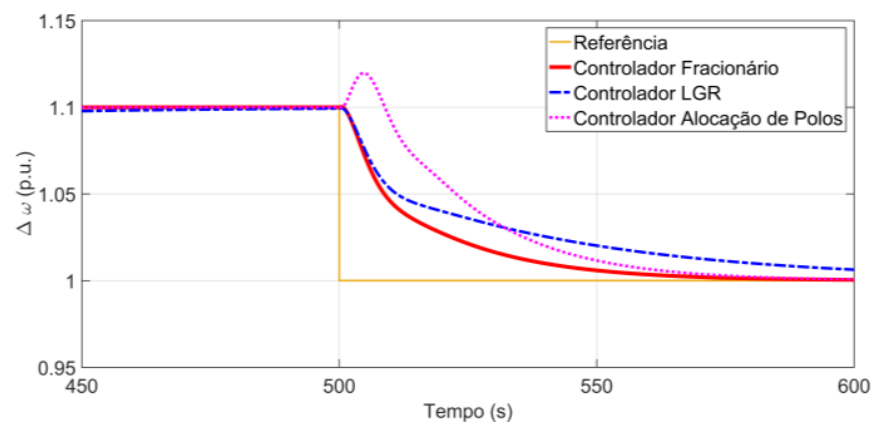

Fig. 5. Variação do sinal de entrada, rejeição de velocidade.

A resposta do controlador para esse teste é apresentada na Fig. 6. Logo, pode-se verificar que assim como a inserção de velocidade ocasiona uma resposta inversa no controlador via alocação de polos, quando essa velocidade é rejeitada, ocorre um sobressinal no instante da variação. O controlador LGR sofre saturação, assim como no primeiro caso. E, o controlador FOPID é o único que possui uma resposta sem variações bruscas.

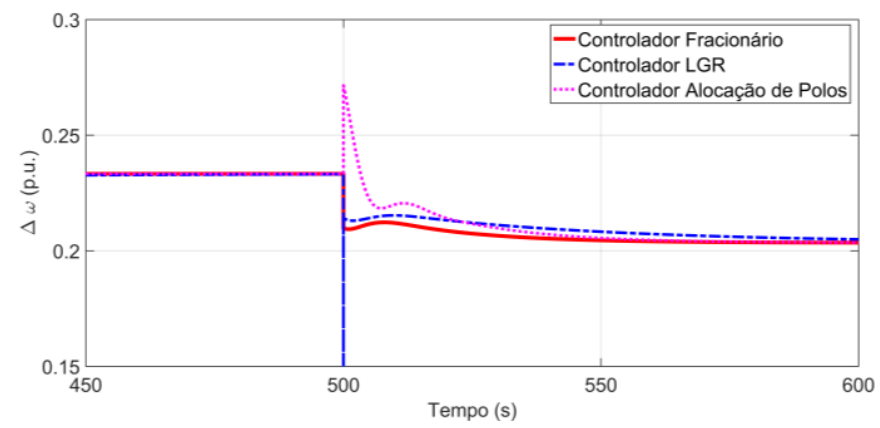

Fig. 6. Resposta do controlador a rejeição de 0,1 p.u de velocidade.

\subsection{Simulação com Inserção de Carga Isolada}

No segundo teste será observado o comportamento dos controladores ao aplicarmos uma variação de carga isolada. Para isso, aplica-se um pulso instantâneo com amplitude de 0,12 p.u., resultando no comportamento da Fig. 7 .

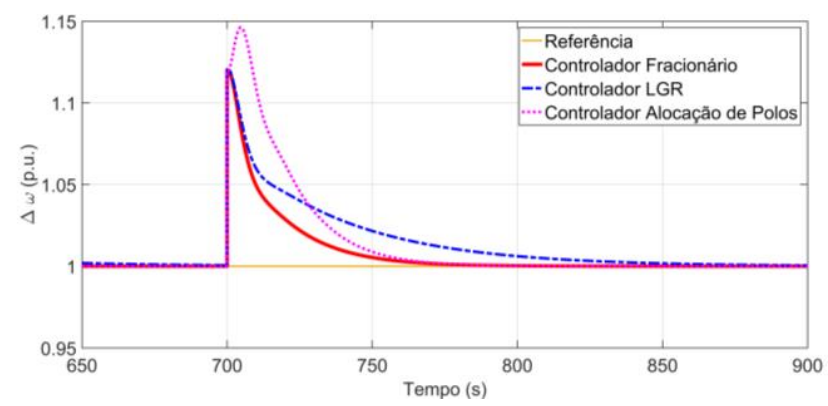

Fig. 7. Inserção de carga

Nesse experimento é possível notar que os controladores, possuem uma resposta rápida e semelhante na inserção da carga. Porém, na rejeição da carga, o controlador por alocação de polos desenvolve um overshoot com maior amplitude de variação e, vale ressaltar que o controlador projetado pela metodologia LGR apresenta o maior tempo de assentamento em comparação as demais metodologias. Pode-se destacar também que o controlador FOPID possui um tempo de acomodação menor que os demais controladores e sem variações bruscas.

Para esse teste, a resposta dos controladores (Fig. 8) possui um resultado semelhante à responda dos controladores quando há a rejeição de velocidade, mostrado na Fig.6.

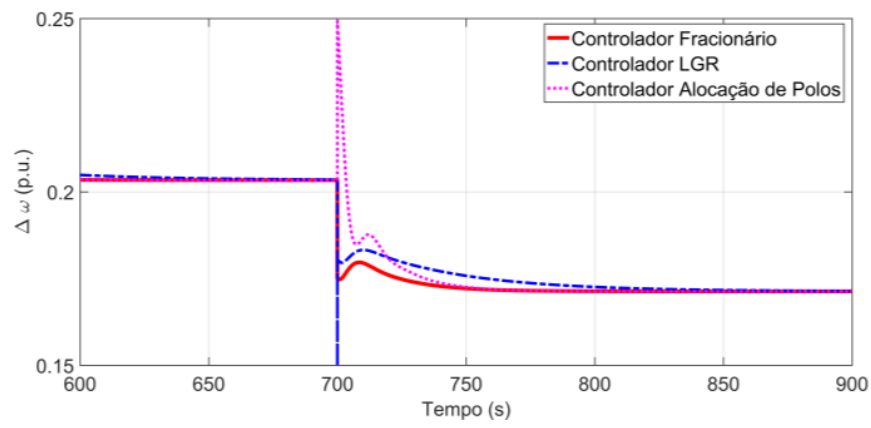

Fig. 8. Resposta dos controladores para Inserção de carga

\section{CONCLUSÕES}

Neste trabalho, foi sintonizado um controlador fracionário FOPID, utilizando o método de sintonia baseado em margens de ganho e fase, aplicado na regulação de velocidade de um sistema de geração em escala reduzida. Objetivando comparar o desempenho dinâmico obtido pelo método fracionário de sintonia, foram sintonizados dois controladores baseados em duas técnicas clássicas de projeto de controladores PID (LGR e alocação polinomial de polos).

Foram realizados testes de variação da referência de velocidade e inserção e retirada de uma carga de 0,12 p.u. Com a inserção do controlador FOPID, pode-se observar o sistema apresentou uma resposta satisfatória em relação ao tempo de acomodação, a não existência de overshoot (máximo sobressinal) e undershoot (resposta inversa).

Comparando as três metodologias, sistema implementado com o controlador FOPID obteve um menor tempo de resposta e menor variação de amplitude, quando comparado aos resultados obtidos pelos controladores projetados baseados nos métodos clássicos. Isso deve-se a flexibilidade obtida no 
projeto do controlador FOPID, pelo fato de obter um total cinco parâmetros de ajuste, em comparação a sua contraparte de ordem inteira, que possui apenas três.

\section{AGRADECIMENTOS}

Esta pesquisa, conforme previsto no Art. 48 do Decreto $\mathrm{n}^{\circ} 6.008 / 2006$, foi financiada pela Samsung Electrônica da Amazônia Ltda, nos termos da Lei Federal n. 8.387/1991, através do convênio $n^{\circ}$ 004, firmado com o CETELI / UFAM.

\section{REFERÊNCIAS}

Ayres Junior, F. A. C, Medeiros, R. L. P., Barra Jr., Barreiros, J. A. L. (2014). Implementação de um regulador de velocidade baseado em lei de controle fracionário aplicado em um sistema de geração de energia de $10 \mathrm{kVA}$. Simpósio Brasileiro de Sistemas Elétricos 2014. Foz do Iguaçu. 2014.

Bazanella, A. S., Silva Jr, J. M. G. (2005). Sistemas de Controle - Princípios e Métodos de Projeto. UFRGS, Porto Alegre.

Farias, N. J. S. (2019). Projeto e Desenvolvimento de um Sistema Emulador de Microgeração Hidráulica de Energia Elétrica. Trabalho de Conclusão de Curso (Bacharelado em Engenharia Elétrica) - UFAM, Amazonas, Brasil.

Finkler, A., Flinker, D. R., Castro, L. S., Milke, T. F. Relação do Crescimento Econômico e Consumo de Energia Elétrica. SALÃO DO CONHECIMENTO UNIJUI, 14, 2016, RS. Ensaio teórico, UNIJUÍ.

Kundur, P. (1994). Power System Stability and Control. McGraw-Hill, pp. 377- 460.

Mathworks. MATLAB. Disponível em: https://www.mathworks.com. Acesso em: 13 fev. 2019.

São Roque, A. A. G. (2015). Aplicação das FPAA na realização de sistemas de ordem fraccionária. Dissertação (Mestrado em Engenharia Eletrotécnica e de Computadores) Instituto Superior de Engenharia do Porto, Portugal.

Monje, A. C., Chen, Y., Vinagre, B. M., Xue, D., Feliu-Battle, V. (2010). Fractional Order Control Systems, Fundamentals and Applications. Springer, Londres.

Nascimento Filho, P. S. (2011). Investigação de Estratégias de Controle Digital para Regulação de Velocidade e Emulação da Dinâmica de Turbinas Hidráulicas, com Implementação e Testes Experimentais em uma Micromáquina de 10 KVA. 2011. Dissertação (Mestrado em Engenharia Elétrica) - UFPA, Pará, Brasil.

Powell, J. D., Franklin, G.F., Emami-Naeini, A. (2013). Sistemas de Controle para Engenharia. 6. ed. Bookman, Porto Alegre.

Rocha, D. C.; Oliveira, F. M. B; Nardi, M. (2012). Simulador de regulador de velocidade para turbinas hidráulicas de usinas hidrelétricas. Trabalho de Conclusão de Curso (Bacharelado em Engenharia Industrial Elétrica) - UTPR, Paraná, Brasil.

Valério, D.; Costa, J. S. (2013). An Introduction to Fractional Control. The Institution of Engineering and Technology, Londres. 